\title{
ON GENERALISED PRONORMAL SUBGROUPS OF FINITE GROUPS
}

\author{
A. BALLESTER-BOLINCHES \\ Departament d'Àlgebra, Universitat de València, 46100 Burjassot, València, Spain \\ e-mail: Adolfo.Ballester@uv.es \\ J. C. BEIDLEMAN \\ Department of Mathematics, University of Kentucky, Lexington, KY 40506-0027, USA \\ e-mail: james.beidleman@uky.edu \\ A. D. FELDMAN \\ Department of Mathematics, Franklin and Marshall College, Lancaster, PA 17604-3003, USA \\ e-mail: afeldman@fandm.edu \\ and M. F. RAGLAND \\ Department of Mathematics, Auburn University Montgomery, Montgomery, AL 36124-4023, USA \\ e-mail: mragland@aum.edu
}

(Received 3 January 2013; accepted 4 November 2013)

\begin{abstract}
For a formation $\mathfrak{F}$, a subgroup $M$ of a finite group $G$ is said to be $\mathfrak{F}$ pronormal in $G$ if for each $g \in G$, there exists $x \in\left\langle U, U^{g}\right\rangle^{\mathfrak{F}}$ such that $U^{x}=U^{g}$. Let $f$ be a subgroup embedding functor such that $f(G)$ contains the set of normal subgroups of $G$ and is contained in the set of Sylow-permutable subgroups of $G$ for every finite group $G$. Given such an $f$, let $f T$ denote the class of finite groups in which $f(G)$ is the set of subnormal subgroups of $G$; this is the class of all finite groups $G$ in which to be in $f(G)$ is a transitive relation in $G$. A subgroup $M$ of a finite group $G$ is said to be $\mathfrak{F}$-normal in $G$ if $G / \operatorname{Core}_{G}(M)$ belongs to $\mathfrak{F}$. A subgroup $U$ of a finite group $G$ is called $\mathrm{K}$ - $\mathfrak{F}$-subnormal in $G$ if either $U=G$ or there exist subgroups $U=U_{0} \leq U_{1} \leq \cdots \leq U_{n}=G$ such that $U_{i-1}$ is either normal or $\mathfrak{F}$-normal in $U_{i}$, for $i=1,2, \ldots, n$. We call a finite group $G$ an $f T_{\mathfrak{F}}$-group if every $\mathrm{K}-\mathfrak{F}$-subnormal subgroup of $G$ is in $f(G)$. In this paper, we analyse for certain formations $\mathfrak{F}$ the structure of $f T_{\mathfrak{F}}$-groups. We pay special attention to the $\mathfrak{F}$-pronormal subgroups in this analysis.
\end{abstract}

2010 Mathematics Subject Classification. 20D10, 20D35, $20 \mathrm{~F} 17$.

1. Introduction and Statements of Results. Note that all groups considered in this paper are finite and we use the notation of [2].

1.1. Extension of pronormality and weak normality. Feldman [9] and Müller [11] independently generalised pronormality of a subgroup of a finite soluble group to $\mathfrak{F}$-pronormality, where $\mathfrak{F}$ is a subgroup-closed saturated formation containing $\mathfrak{N}$, the class of all nilpotent groups. Both used the concept of an $\mathfrak{F}$-base, a generalisation of a 
Hall system of a soluble group. But $\mathfrak{F}$-bases cannot be defined for non-soluble groups, so we use the following definition due to Müller.

Definition 1. Let $G$ be a group and $U$ be a subgroup of $G$. Then $U$ is said to be $\mathfrak{F}$-pronormal in $G$ if, for each $g \in G$, there exists $x \in\left\langle U, U^{g}\right\rangle^{\mathfrak{F}}$ such that $U^{x}=U^{g}$.

See the remark after [4, Definition 2] for a brief proof that $\mathfrak{N}$-pronormality is simply pronormality so that this is a legitimate generalisation.

It is easy to see that when $\mathfrak{F}$ contains $\mathfrak{N}$, every $\mathfrak{F}$-pronormal subgroup is pronormal. This immediately leads to the question of how to characterise groups $G$ such that a subgroup is $\mathfrak{F}$-pronormal in $G$ if and only if it is pronormal in $G$. In a soluble case, Müller [11] showed that for $\mathfrak{F}$ saturated and containing $\mathfrak{N}$, the class of groups whose maximal subgroups are all $\mathfrak{F}$-pronormal is the same as the class of groups whose $\mathfrak{F}$ normalisers are nilpotent if and only if the latter class is a saturated formation [11, 7.3]. The more general question is quite difficult, so a good first step is to look at special cases like the $f T_{\mathfrak{F}}$-groups defined below, where $\mathfrak{F}$ is a formation of finite groups containing $\mathfrak{N}$ with certain other properties that are weaker than those assumed in $[\mathbf{9}$, 11].

Another interesting subgroup embedding property is weak normality.

Definition 2. A subgroup $H$ of $G$ is weakly normal in $G$ if for $g \in G,\left\langle H, H^{g}\right\rangle \leq$ $N_{G}(H)$ implies $g \in N_{G}(H)$.

It is easy to see that pronormal subgroups are weakly normal $[2,1.5 .9]$. A nice property of weak normality is that the join of two pronormal subgroups is necessarily weakly normal $[2,1.5 .12]$, but not necessarily pronormal $[2,1.5 .11]$. Thus, it makes sense to generalise weak normality in a way analogous to the way we generalise pronormality.

DEFINITION 3. If $\mathfrak{F}$ is a formation, a subgroup $H$ of $G$ is $\mathfrak{F}$-weakly normal in $G$ if for $g \in G,\left\langle H, H^{g}\right\rangle^{\mathfrak{F}} \leq N_{G}(H)$ implies $g \in N_{G}(H)$.

We will see below that weak $\mathfrak{N}$-normality is the same as weak normality, and that therefore our $\mathfrak{F}$-weakly normal subgroups are weakly normal, enabling us to incorporate $\mathfrak{F}$-weak normality into some of our generalised results.

We will see below that $\mathfrak{F}$-pronormal subgroups are $\mathfrak{F}$-weakly normal, and that the join of $\mathfrak{F}$-pronormal subgroups is $\mathfrak{F}$-weakly normal when $\mathfrak{F}$ is subgroup-closed.

1.2. $f T$-groups. A group $G$ is said to be a $T$-group if every subnormal subgroup of $G$ is normal in $G$, a $P T$-group if every subnormal subgroup of $G$ is permutable in $G$ and a $P S T$-group if every subnormal subgroup of $G$ is Sylow-permutable $(S$ permutable) in $G$. Let $f$ be a subgroup embedding functor contained in the functor $s n$ of subnormal subgroups and containing the functor $n$ of normal subgroups. As in [3], if $X$ is a subgroup of group $G$ belonging to $f(G)$, we write $X f G$. Prominent examples for $f$ include $n$; $p$, where $p(G)$ is the set of permutable subgroups of $G$; and $p S$, where $p S(G)$ is the class of $S$-permutable subgroups of $G$. We may then define an $f T$-group to be a group such that $X s n G$ implies $X f G$ so that the $T$-, $P T$-and $P S T$-groups are examples of $f T$-groups, where $f$ is, respectively, $n, p$ and $p S$. Let $f W$ be the class of groups $G$ such that $H \leq G$ implies $H f G$; clearly, $f W$ is a subclass of $f T$. For $f=n, p$ and $p S$, we have that $f W$ is a class of Dedekind groups, Iwasawa groups and nilpotent groups respectively. 
1.3. Extension of subnormality. Let $\mathfrak{F}$ be a formation. A subgroup $M$ of a group $G$ is said to be $\mathfrak{F}$-normal in $G$ if $G / \operatorname{Core}_{G}(M)$ belongs to $\mathfrak{F}{ }^{1}$ It is clear that $M$ is $\mathfrak{F}$-normal if and only if $G^{\mathfrak{F}}$, the $\mathfrak{F}$-residual of $G$, is contained in $M$. Kegel (see [3, 6.1.4]) introduced an extension of subnormality, which has come to be known as K-F-subnormality.

Definition 4. A subgroup $U$ of a group $G$ is called a K-F-subnormal subgroup of $G$ if either $U=G$ or there exist subgroups

$$
U=U_{0} \leq U_{1} \leq \cdots \leq U_{n}=G
$$

such that $U_{i-1}$ is either normal or $\mathfrak{F}$-normal in $U_{i}$, for $i=1,2, \ldots, n$.

It is not difficult to prove that the subnormal subgroups of a group $G$ are exactly the K-Ni-subnormal subgroups. For a summary of results concerning K-F-subnormality see [3, Chapter 6].

1.4. Statements of results. Hereafter assume that $f$ is a subgroup embedding functor such that $f$ contains $n$ and is contained in $p S$; if $H f G$ and $N$ is normal in $G$, then $H N / N f G / N$; if $N$ is normal in $G$ and $H / N f G / N$, then $H f G$; and if $H \leq L s n$ $G$ and $H f G$, then $H f L$.

Thus, $n, p$ and $p S$ are all possibilities for $f$. Note that for any of our possible $f$ 's, the class of $f T$-groups will be closed under epimorphic images and subnormal subgroups.

DEFINITION 5. Let $\mathfrak{F}$ be a formation. A group $G$ is said to be an $f T_{\mathfrak{F}}$-group if $H f G$ for every K-F-subnormal subgroup $H$ of $G$.

As in [1], we see that for every $f$ under consideration, $f T_{\mathfrak{F}}$ is a class of $f T$-groups, and $f T_{\mathfrak{F}}$ is closed under the taking of subnormal subgroups. Moreover, $n T_{\mathfrak{N}}=n T=$ $T, p T_{\mathfrak{N}}=p T=P T$ and $p S T_{\mathfrak{N}}=p S T=P S T$. Because $f$ is contained in $p S, H f G$ implies $H$ sn $G$, every $f T$-group is a $P S T$-group and $f W$ is always a subclass of $\mathfrak{N}$.

In the results below, if we are referring simultaneously to more than one $f$, we will normally use the general notation, but if we are referring to a specific $f$, we will usually employ the standard notation. Thus, for example, we will generally use PST rather than $f T$ or $p S T$.

Let $\mathfrak{O}$ be the set of ordered pairs $(p, q)$, where $p$ and $q$ are prime numbers such that $q$ divides $p-1$, and for $(p, q)$ in $\mathfrak{O}$, denote by $X_{(p, q)}$ a non-abelian group of order $p q$. Then, let $\mathfrak{X}$ be the class consisting of every group that is isomorphic to $X_{(p, q)}$ for some $(p, q) \in \mathfrak{O}$. Furthermore, denote by $\mathfrak{X}_{\mathfrak{F}}$ the class $\mathfrak{X} \cap \mathfrak{F}$. Finally, let $\mathfrak{Y}$ be the class of non-abelian finite simple groups, and let $\mathfrak{Y} \mathfrak{F}$ be the class $\mathfrak{Y} \cap \mathfrak{F}$.

We say a formation $\mathfrak{F}$ possesses property $\alpha$ if $\mathfrak{F}$ is closed under taking subnormal $\mathfrak{X}$-subgroups of soluble PST-groups. As seen in [1, Remark 1.4], every saturated formation containing the class of all nilpotent groups has property $\alpha$. For the sake of completeness, we include this result and its proof below as Lemma 1.

Definition 6. If $\mathfrak{F}$ is a formation, a group $G$ is said to be an $R_{\mathfrak{F}}$-group if

(i) No section of $G / G^{\mathfrak{S}}$ is isomorphic to an element of $\mathfrak{X}_{\mathfrak{F}}$.

(ii) No chief factor of $G^{\mathfrak{S}}$ is isomorphic to an element of $\mathfrak{Y}_{\mathfrak{F}}$.

$\overline{{ }^{1} \text { Note that }}$ in other references, $\mathfrak{F}$-normality is defined in a slightly different way. 
Here $G^{\mathfrak{S}}$ denotes the residual of $G$ with respect to the formation $\mathfrak{S}$ of all soluble groups.

Let $f R_{\mathfrak{F}}=f T \cap R_{\mathfrak{F}}$. Note that if $f_{1}$ is contained in $f_{2}$ so that $f_{1} T$ is contained in $f_{2} T$, then $f_{1} R_{\mathfrak{F}}=f_{1} T \cap f_{2} R_{\mathfrak{F}}$.

We will make use of the following, a principal result from our previous work:

THEOREM 1. [1, Theorem 1.8] If $G$ is a group, $f$ is a subgroup embedding functor, and $\mathfrak{F}$ is a formation that contains $\mathfrak{N}$ and has property $\alpha$, then

(i) $f T_{\mathfrak{F}} \cap \mathfrak{S}=f R_{\mathfrak{F}} \cap \mathfrak{S}$.

(ii) If PST $\cap \mathfrak{F}$ is closed under taking subnormal non-abelian simple subgroups, then $f T_{\mathfrak{F}}=f R_{\mathfrak{F}}$.

COROLlaRY 1. For each formation $\mathfrak{F}$ that contains $\mathfrak{N}$ and has property $\alpha$, if the class $f T \cap \mathfrak{S}$ is subgroup-closed (as it is for $f=n, p$ and $p S$ ), then the class $f T_{\mathfrak{F}} \cap \mathfrak{S}$ is subgroup-closed, as well.

We will also make use of the following.

THEOREM 2. [1, Theorem 1.10] Let $\mathfrak{F}$ be a formation containing $\mathfrak{N}$ and $f=n, p$ or $p S$. Then $G$ is a soluble $f T_{\mathfrak{F}^{-}}$group if and only if $G$ satisfies:

(i) $G^{\mathfrak{F}}$ is a normal abelian Hall subgroup of $G$ with odd order;

(ii) $X / X^{\mathfrak{F}}$ is an $f W$-group for every $X$ sn $G$;

(iii) every subgroup of $G^{\mathfrak{F}}$ is normal in $G$.

COROLlaRY 2. Let $G$ be a soluble group and $\mathfrak{F}$ be a formation containing $\mathfrak{N}$, and $f=n$, $p$ or $p S$. Then $G$ is an $f T_{\mathfrak{F}}$-group if and only if $G$ is a PST-group and $X / X^{\mathfrak{F}} \in f W$ for every subnormal subgroup $X$ of $G$.

COROLlARY 3. Let $G$ be a group and $\mathfrak{F}$ be a formation such that $\mathfrak{F}$ contains $\mathfrak{N}$ and has property $\alpha$, and $f T \cap \mathfrak{S}$ is subgroup-closed (as it is for $f=n, p$ and $p S$ ). If $G$ is a soluble $f T_{\mathfrak{F}}$-group, then $H$ is pronormal in $G$ if and only if $H$ is $\mathfrak{F}$-pronormal in $G$.

The definition below allows us to expand somewhat the generalisation of the results of Peng [12], Robinson [13] and Feldman [10], characterising soluble $T$-groups and $T_{\mathfrak{F}}$-groups that appear as [4, Theorem 1].

THEOREM 3. Let $G$ be a group and $\mathfrak{F}$ be a formation that contains $\mathfrak{N}$ and has property $\alpha$. The following statements are pairwise equivalent:

(i) $G$ is a soluble $T_{\mathfrak{F}}$-group.

(ii) Every subgroup of $G$ is $\mathfrak{F}$-pronormal in $G$.

(iii) Every subgroup of $G$ is $\mathfrak{F}$-weakly normal in $G$.

DEFINITION 7. If $\mathfrak{F}$ is a formation, a subgroup $H$ of $G$ is $\mathfrak{F}$-weakly Frattini in $G$ if whenever $H \leq L$ and $L$ is K-F-subnormal in $G$, then $N_{G}(H) L=G$.

When $\mathfrak{F}=\mathfrak{N}$, this definition is equivalent to the usual definition of satisfying the weak Frattini argument, which is sometimes simply called the Frattini argument. It is not difficult to prove, using induction, that '( $\mathfrak{N})$-subnormal' can be replaced without loss by 'normal' in that traditional Frattini argument.

This definition allows for a very easy result that applies to non-soluble as well as soluble groups, and an equivalence that applies only to soluble groups.

THEOREM 4. Let $G$ be a group and $\mathfrak{F}$ be a formation. 
(i) If every subgroup of $G$ is weakly $\mathfrak{F}$-Frattini in $G$, then $G$ is a $T_{\mathfrak{F}}$-group.

(ii) If $\mathfrak{F}$ contains $\mathfrak{N}$ and has property $\alpha$ and $G$ is soluble, then $G$ is a $T_{\mathfrak{F}}$-group if and only if every subgroup of $G$ is weakly $\mathfrak{F}$-Frattini in $G$.

A group satisfies $\mathbf{H}_{p}$ if every normal subgroup of a Sylow $p$-subgroup of $G$ is pronormal in $G$. By [2, Lemma 2.2.22], if $G$ is a $p$-soluble group that satisfies $\mathbf{H}_{p}$, and $N$ is a normal subgroup of $G$, then $G / N$ satisfies $\mathbf{H}_{p}$. Also, a group $G$ is a soluble $P S T$-group if and only if every subgroup of $G$ satisfies $\mathbf{H}_{p}$ for all primes $p$ [2, Corollary 2.2.24, Theorem 2.2.9]. Furthermore, [2, Theorems 2.2.25, 2.2.3], a group $G$ is a soluble $P T$-group if and only if it satisfies $\mathbf{H}_{p}$ and has Iwasawa Sylow $p$-subgroups for all primes $p$. Similarly [2, Theorems 2.2.25, 2.2.2, 2.2.3], a group $G$ is a soluble $T$-group if and only if it satisfies $\mathbf{H}_{p}$ and has Dedekind Sylow $p$-subgroups for all primes $p$.

We generalise $\mathbf{H}_{p}$ as follows: A group $G$ satisfies $\mathbf{H}_{p \mathfrak{F}}$ if every normal subgroup of a Sylow $p$-subgroup of $G$ is $\mathfrak{F}$-pronormal in $G$. For the groups and formations under consideration here, every $\mathfrak{F}$-pronormal subgroup of $G$ is pronormal in $G$, so a group that satisfies $\mathbf{H}_{p \mathfrak{F}}$ also satisfies $\mathbf{H}_{p}$. With this definition, we can obtain the following results.

THEOREM 5. Let $G$ be a group and $\mathfrak{F}$ be a formation that contains $\mathfrak{N}$ and has property $\alpha$. Then $G$ is a soluble PST $T_{\mathfrak{F}}$-group if and only if every subgroup of $G$ satisfies $\mathbf{H}_{p \mathfrak{F}}$ for all primes $p$.

Corollary 4. Let $G$ be a group and $\mathfrak{F}$ be a formation that contains $\mathfrak{N}$ and has property $\alpha$. If $G$ is a soluble PST-group, it is a PST $T_{\mathfrak{F}}$-group if and only if in every subgroup $L$ of $G$, every pronormal subgroup of $L$ is $\mathfrak{F}$-pronormal in $L$.

COROllary 5. Let $G$ be a group and $\mathfrak{F}$ be a formation that contains $\mathfrak{N}$ and has property $\alpha$.

(i) $G$ is a soluble $P T_{\mathfrak{F}}$-group if and only if (every subgroup of) $G$ satisfies $\mathbf{H}_{p \mathfrak{F}}$ and $G$ has Iwasawa Sylow p-subgroups for all primes $p$.

(ii) $G$ is a soluble $T_{\mathfrak{F}}$-group if and only if (every subgroup of) $G$ satisfies $\mathbf{H}_{p \mathfrak{F}}$ and $G$ has Dedekind Sylow p-subgroups for all primes $p$.

Note that Theorem 5 and Corollary 5 imply that if $\mathfrak{F}$ has Property $\alpha$, then $G$ is a soluble $P T_{\mathfrak{F}}$-group ( $T_{\mathfrak{F}}$-group) if and only if $G$ is a soluble $P S T_{\mathfrak{F}}$-group and for all $p$, $G$ has Iwasawa (Dedekind) Sylow $p$-subgroups.

Corollary 6. Let $G$ be a group and $\mathfrak{F}$ be a formation that contains $\mathfrak{N}$ and has property $\alpha$.

(i) If $G$ is a soluble PT-group, it is a $P T_{\mathfrak{F}}$-group if and only if in (every subgroup $L$ of) $G$, every pronormal subgroup (of $L$ ) is $\mathfrak{F}$-pronormal (in $L$ ).

(ii) If $G$ is a soluble $T$-group, it is a $T_{\mathfrak{F}}$-group if and only if in (every subgroup $L$ of) $G$, every pronormal subgroup (of $L$ ) is $\mathfrak{F}$-pronormal (in $L$ ).

We say, a group $G$ satisfies $f T_{p}$, where $p$ is a prime number, if $H f G$ for every subnormal $p^{\prime}$-perfect subgroup $H$ of $G$. Thus, clearly any group contained in $f T$ is contained in $f T_{p}$ for all $p$. In the literature, $n T_{p}$ is denoted as $T_{p}, p T_{p}$ is denoted as $T_{p}^{\prime}$, and $p S T_{p}$ is denoted as $T_{p}^{\prime \prime}$. The following result combines works by Bryce and Cossey [7] and Beidleman and Heineken [6].

THEOREM 6. Let $f=n$, $p$ or $p S$. If $G$ is a soluble group, then $G \in f T$ if and only if $G \in f T_{p}$ for all primes $p$. 
We can generalise $f T_{p}$ in the obvious way: We say $G$ satisfies $f T_{p \mathfrak{F}}$ if $H f G$ for every K-F-subnormal $p^{\prime}$-perfect subgroup $H$ of $G$. Thus, $f T_{p \mathfrak{N}}$ is just $f T_{p}$ for each of our $f$ 's.

The natural extension of the above theorem is as follows.

THEOREM 7. Let $\mathfrak{F}$ be a formation that contains $\mathfrak{N}$ and has property $\alpha$, where $f=n$, p or $p S$. If $G$ is a soluble group, then $G \in f T_{\mathfrak{F}}$ if and only if $G \in f T_{p \mathfrak{F}}$ for all primes $p$.

\section{Preliminaries.}

LEMMA 1. Every saturated formation $\mathfrak{F}$ containing $\mathfrak{N}$ has property $\alpha$.

Proof. Let $\mathfrak{F}=L F(F)$ be a saturated formation locally defined by the integrated and full formation function $F$. We prove that $\mathfrak{F}$ has property $\alpha$. Assume that $G$ is a soluble $P S T$-group belonging to $\mathfrak{F}$, and let $H$ be a subnormal $X_{(p, q)}$-subgroup of $G$ for some $(p, q) \in \mathfrak{O}$. Suppose first that $G$ is a primitive group. Then $N=\operatorname{Soc}(G)$ is a self-centralising minimal normal subgroup of $G$ which is complemented by a core-free maximal subgroup $S$ of $G$. According to [2, Theorem 2.1.8], $N$ is a Sylow subgroup of $G$, and $S$ is a nilpotent Hall subgroup of $G$. Since $H$ is a non-trivial subnormal subgroup of $G$, it follows that $N$ is a $p$-group. Then $N$ is of order $p$ and $N$ is contained in $H=$ $N(H \cap S)$. Since $G \in \mathfrak{F}$, we have that $S \in F(p)$ and so $H \cap S \in F(p)$ by [3, Theorem 2.1.8]. Hence, $H \in \mathfrak{S}_{p} F(p)=F(p) \subseteq \mathfrak{F}$. Assume that $G$ is an arbitrary soluble $P S T$ group. If $S$ is a maximal subgroup of $G$, then $G / \operatorname{Core}_{G}(S)$ is a primitive soluble group and $H$ Core $_{G}(S) / \operatorname{Core}_{G}(S)$ is either a subgroup of $G / \operatorname{Core}_{G}(S)$ of prime power order or $H \operatorname{Core}_{G}(S) / \operatorname{Core}_{G}(S)$ is a subnormal $\mathfrak{X}$-subgroup of $G / \operatorname{Core}_{G}(S)$. In any case, $H \operatorname{Core}_{G}(S) / \operatorname{Core}_{G}(S) \in \mathfrak{F}$ by the above argument. Therefore, $H /(H \cap \Phi(G)) \in \mathfrak{F}$. By [5, Theorem 3.1], $H \in \mathfrak{F}$.

The following lemmas will be used in the proofs of our main results.

LEMMA 2. Suppose $\mathfrak{F}$ is a formation containing $\mathfrak{N}$.

(i) If $G \in f R_{\mathfrak{F}}$ and $K$ is normal in $G$, then $G / K \in f R_{\mathfrak{F}}$; i.e. $f R_{\mathfrak{F}}$ is closed under epimorphic images.

(ii) Suppose $\mathcal{F}$ has property $\alpha$ and $P S T \cap \mathfrak{F}$ is closed under taking subnormal nonabelian simple subgroups. If $G \in f R_{\mathfrak{F}} \cap \mathfrak{F}$, then $G \in f W$.

(iii) Suppose $H \leq G \in f R_{\mathfrak{F}} \cap \mathfrak{S}$. If $H$ sn $G$ or $f T \cap \mathfrak{S}$ is subgroup-closed, then $H \in$ $f R_{\mathfrak{F}}$.

(iv) If $H$ is a subgroup of $G$ with $G^{\mathfrak{F}} \leq H$, then $H$ is $K$-F-subnormal in $G$. In particular, if $G$ is an $\mathfrak{F}$-group, then every subgroup of $G$ is $K$-F-subnormal in $G$. Hence, $G \in f T_{\mathfrak{F}} \cap \mathfrak{F}$ implies $G \in f W$.

Proof. For (i), let $G \in f R_{\mathfrak{F}}$, and let $K$ be a normal subgroup of $G$. Then $(G / K)^{\mathfrak{S}}=$ $G^{\mathfrak{S}} K / K$, so $(G / K) /(G / K)^{\mathfrak{S}}=(G / K) /\left(G^{\mathfrak{S}} K / K\right)$ is isomorphic to $G / G^{\mathfrak{S}} K$, which is an epimorphic image of $G / G^{\mathfrak{S}}$, and therefore has no section that is an element of $\mathfrak{X}_{\mathfrak{F}}$. Similarly, $G^{\mathfrak{S}} K / K$ is isomorphic to an epimorphic image of $G^{\mathfrak{S}}$, so it has no chief factor that is an element of $\mathfrak{Y}_{\mathfrak{F}}$. Since $G / K \in f T, G / K \in f R_{\mathfrak{F}}$.

Suppose that (ii) is false and let $G$ be a non- $f W$-group of minimal order in $f R_{\mathfrak{F}} \cap \mathfrak{F}$. Suppose $N$ is a minimal normal subgroup of $G$. Hence, $G / N \in \mathfrak{F}$, and $G / N \in f R_{\mathfrak{F}}$ by (i) above. Thus, $G / N$ is in $f W$ by minimality of $G$. Now if $N \cap G^{\mathfrak{S}}=1$, then $N$ is isomorphic to a subgroup of $G / G^{\mathfrak{S}}$ and must be abelian. This means that if $N$ is non-abelian, $N \leq G^{\mathfrak{S}}$. Furthermore, since $G$ is an $f T$-group, it is a $P S T$-group, so $N$ 
is non-abelian simply by [2, Proposition 2.1.1], so it is a chief factor of $G^{\mathfrak{S}}$. However, $G \in P S T \cap \mathfrak{F}$, so $N \in \mathfrak{F}$ by hypothesis. This contradicts the assumption that $G \in f R_{\mathfrak{F}}$. Thus, $N$ must be abelian; since $G / N$ is nilpotent, $G$ is soluble and $G^{\mathfrak{S}}=1$. And since $G$ is a $P S T$-group, $|N|$ is some prime $p$. If $N$ is central in $G$, then $G$ is nilpotent and therefore in $f W$ since it is an $f T$-group, so we may assume $N$ is not central. Since $\mathfrak{N}$ is saturated, $N$ is a unique minimal normal subgroup of $G$, and $N \not \subset \Phi(G)$, so $N$ is complemented by a maximal core-free subgroup $M$, with $C_{M}(N)=1$. Let $q$ be a prime dividing $|M|$ different from $p$, and let $x$ be an element of $Q$ of order $q$. Then $N\langle x\rangle$ is isomorphic to $X_{(p, q)}$ and is subnormal in $G$ because $G / N$ is nilpotent. Hence, $N\langle x\rangle \in \mathfrak{F}$ by property $\alpha$, contradicting the assumption that $G \in f R_{\mathfrak{F}}$ and establishing the result.

For (iii), we observe that $G^{\mathfrak{S}}$ and $H^{\mathfrak{S}}$ are trivial, so condition (ii) of Definition 6 is trivial. Since the sections of $H$ are the sections of $G$, condition (i) holds in $H$ because it holds in $G$. To see that $H \in f T$, observe that all our $f$ 's are closed under the taking of subnormal subgroups. Note that by [2, Corollaries 2.1.9, 2.1.13], the classes of soluble $T$-, $P T$ - and $P S T$-groups are all subgroup-closed.

For condition (iv), see [4, Lemma 1(iv)].

In the lemma below, all but condition (iv) appear in [11].

Lemma 3. See [4, Lemma 3]. Let $U$ be a subgroup of a group $G$ and let $\mathfrak{F}$ be a formation.

(i) If $U \leq H$ and $U$ is $\mathfrak{F}$-pronormal in $G$, then $U$ is $\mathfrak{F}$-pronormal in $H$.

(ii) If $N$ is a normal subgroup of $G$ and $U$ is $\mathfrak{F}$-pronormal in $G$, then $U N / N$ is F-pronormal in $G / N$.

(iii) If $N$ is a normal subgroup of $G$ and $U / N$ is $\mathfrak{F}$-pronormal in $G / N$, then $U$ is $\mathfrak{F}$-pronormal in $G$.

(iv) If $U$ is maximal, $\mathfrak{F}$-pronormal and $K$-F-subnormal in $G$, then $U$ is normal in $G$. In particular, if $G \in \mathfrak{F}$ and $U$ is maximal and $\mathfrak{F}$-pronormal in $G$, then $U$ is normal in $G$.

Proof. For condition (iv), let $g$ be an element of $G$ so that $\left\langle U, U^{g}\right\rangle$ is equal to either $U$ or $G$. Now $U^{\mathfrak{F}} \leq U$, and if $U^{g} \neq U$, then $U$ is not normal in $G$, so it is $\mathfrak{F}$-normal in $G$ and therefore $G^{\mathfrak{F}} \leq U$. Thus, there exists $x \in U$ such that $U^{x}=U^{g}$ because $U$ is $\mathrm{K}$-F-pronormal. Hence, $U^{g}=U$ as claimed. For the particular case, note that every subgroup of a member of $\mathfrak{F}$ is $\mathrm{K}$-F-normal and hence $\mathrm{K}$-F-subnormal.

Proposition 1. [4, Proposition 1] Let $U$ be a subgroup of a group $G$ and let $N$ be a normal subgroup of $G$ such that $U \leq N \leq G$. Then, if $\mathfrak{F}$ is a formation, the following conditions are equivalent:

(i) $U$ is $\mathfrak{F}$-pronormal in $G$.

(ii) $U$ is $\mathfrak{F}$-pronormal in $N$ and $G=N_{G}(U) N$.

In [2, Theorem 2.3.1], the connection between $T$-groups and weak normality is established. With the following result, we see that, because $\mathfrak{N}$-weak normality is a simply weak normality, when our Theorem 3 connects $T_{\mathfrak{F}}$-groups with $\mathfrak{F}$-weak normality, it is generalising that theorem.

LEMMA 4. If $H$ is a subgroup of $G$, the following conditions are equivalent:

(i) $H$ is weakly normal in $G$.

(ii) $H<\left\langle H, H^{g}\right\rangle$ implies $N_{\left\langle H, H^{g}\right\rangle}(H)$ is a proper subgroup of $\left\langle H, H^{g}\right\rangle$. 
(iii) $H<\left\langle H, H^{g}\right\rangle$ implies $N_{\left\langle H, H^{g}\right\rangle}(H)$ is a proper self-normalising subgroup of $\left\langle H, H^{g}\right\rangle$.

(iv) $H$ is $\mathfrak{N}$-weakly normal in $G$.

Proof. First we see that (i) implies (ii). A subgroup $H$ of $G$ is weakly normal in $G$ if whenever $H^{g}$ normalises $H$, then $g \in N_{G}(H)$. Hence, if $H$ is weakly normal and proper in $\left\langle H, H^{g}\right\rangle$, the latter assumption implies $g \notin N_{G}(H)$, and the former implies $H^{g}$ does not normalise $H$. The desired conclusion follows immediately.

To show that (ii) implies (iii), we only need to show that (ii) implies that $N_{\left\langle H, H^{g}\right\rangle}(H)$ is self-normalising in $\left\langle H, H^{g}\right\rangle$. So suppose not; then there exists an element $x$ of $\left\langle H, H^{g}\right\rangle$ that normalises $N_{\left\langle H, H^{g}\right\rangle}(H)$ but does not normalise $H$. Hence, $H<\left\langle H, H^{x}\right\rangle$. But $H \leq$ $N_{\left\langle H, H^{g}\right\rangle}(H)$ implies that $H^{x} \leq N_{\left\langle H, H^{g}\right\rangle}(H)$, so $\left\langle H, H^{x}\right\rangle$ normalises $H$, contradicting (ii).

To show that (iii) implies (iv), suppose $\left\langle H, H^{g}\right\rangle^{\mathfrak{N}}$ normalises $H$ but $g \notin N_{G}(H)$, so $H<\left\langle H, H^{g}\right\rangle$. Then $\left\langle H, H^{g}\right\rangle^{\mathfrak{N}} \leq N_{\left\langle H, H^{g}\right\rangle}(H) \leq\left\langle H, H^{g}\right\rangle$. Thus, $N_{\left\langle H, H^{g}\right\rangle}(H) /\left\langle H, H^{g}\right\rangle^{\mathfrak{N}}$, being a subgroup of the nilpotent group $\left\langle H, H^{g}\right\rangle /\left\langle H, H^{g}\right\rangle^{\mathfrak{N}}$, is subnormal in $\left\langle H, H^{g}\right\rangle /\left\langle H, H^{g}\right\rangle^{\mathfrak{N}}$, so $N_{\left\langle H, H^{g}\right\rangle}(H)$ is subnormal in $\left\langle H, H^{g}\right\rangle$. Now no subnormal subgroup can be both self-normalising and proper, so this contradicts (iii).

Finally, suppose $H$ is $\mathfrak{N}$-weakly normal in $G$ and $\left\langle H, H^{g}\right\rangle$ normalises $H$. Then $\left\langle H, H^{g}\right\rangle^{\mathfrak{N}}$ normalises $H$, so $g \in N_{G}(H)$ by hypothesis, establishing the claim.

Note that if $\mathfrak{F}$ is a formation containing $\mathfrak{N}$, and $H$ is $\mathfrak{F}$-weakly normal in $G$, then $H$ is $\left(\mathfrak{N}\right.$-)weakly normal in $G$ because $\left\langle H, H^{g}\right\rangle^{\mathfrak{F}}$ is contained in $\left\langle H, H^{g}\right\rangle^{\mathfrak{N}}$. In this sense, $\mathfrak{F}$-weak normality resembles $\mathfrak{F}$-pronormality; the following result further explores this similarity.

LeMma 5. Let $\mathfrak{F}$ be a formation. If $H$ is $\mathfrak{F}$-pronormal in $G$, then $H$ is $\mathfrak{F}$-weakly normal in $G$.

Proof. Suppose $H \leq G$ and $\left\langle H, H^{g}\right\rangle^{\mathfrak{F}} \leq N_{G}(H)$ for some $g \in G$. If $H$ is $\mathfrak{F}$ pronormal in $G$, then there exists $x \in\left\langle H, H^{g}\right\rangle^{\mathfrak{F}}$ such that $H^{x}=H^{g}$. But then $x \in N_{G}(H)$, so $H^{g}=H$ and $g \in N_{G}(H)$; thus $H$ is $\mathfrak{F}$-weakly normal in $G$.

LEMMA 6. If $U$ is maximal, $\mathfrak{F}$-weakly normal and $K$-F-subnormal in $G$, then $U$ is normal in $G$. In particular, if $G \in \mathfrak{F}$ and $U$ is maximal in $G$ and $\mathfrak{F}$-weakly normal in $G$, then $U$ is normal in $G$.

Proof. As in the proof of Lemma 3(iv), for all $g \in G,\left\langle U, U^{g}\right\rangle^{\mathfrak{F}} \leq U \leq N_{G}(U)$, so $g \in N_{G}(U)$ by definition of $\mathfrak{F}$-weakly normal. The particular case follows as in Lemma 3(iv).

Note that together, Lemmas 5 and 6 imply Lemma 3(iv).

To ensure that conditions (ii) and (iii) of Theorem 3 are worth distinguishing between, we need to show that the converse of Lemma 5 is not true. Thus, we need an example of a group $G$ and a subgroup $H$ such that $H$ is $\mathfrak{F}$-weakly normal in $G$ but not $\mathfrak{F}$-pronormal in $G$. Ballester-Bolinches et al. [2, Example 1.5.10] present a group that has a subgroup of order 21 and index 14 that is $(\mathfrak{N}$-)weakly normal but not $(\mathfrak{N}$-)pronormal.

To conclude this section, we note that in the case of a subgroup-closed formation $\mathfrak{F}$, we can generalise the above-mentioned result that the join of pronormal subgroups is weakly closed.

LEMMA 7. Suppose $\mathfrak{F}$ is a subgroup-closed formation and $A$ and $B$ are $\mathfrak{F}$-pronormal in $G$. Then $\langle A, B\rangle$ is $\mathfrak{F}$-weakly normal in $G$. 
Proof. Let $J=\langle A, B\rangle$ and if $g \in G$, let $C=\left\langle A, A^{g}\right\rangle, D=\left\langle B, B^{g}\right\rangle$ and $X=\left\langle J, J^{g}\right\rangle$. Then $C / C \cap X^{\mathfrak{F}}$, being isomorphic to a subgroup of the $\mathfrak{F}$-subgroup $X / X^{\mathfrak{F}}$, is itself in $\mathfrak{F}$ by hypothesis. Hence, $C^{\mathfrak{F}} \leq X^{\mathfrak{F}}$, and similarly, $D^{\mathfrak{F}} \leq X^{\mathfrak{F}}$. Now suppose $X^{\mathfrak{F}}=$ $\left\langle J, J^{g}\right\rangle^{\mathfrak{F}} \leq N_{G}(J)$. Then because $A$ is $\mathfrak{F}$-pronormal in $G$, there exists $c \in C^{\mathfrak{F}}$ such that $A^{g}=A^{c}$; similarly, there exists $d \in D^{\mathfrak{F}}$ such that $B^{g}=B^{d}$. But then as seen above, $c$ and $d$ are in $X^{\mathfrak{F}}$, so $c$ and $d$ are in $N_{G}(J)$. Hence, $J^{g}=\left\langle A, B, A^{g}, B^{g}\right\rangle=\left\langle A, B, A^{c}, B^{d}\right\rangle \leq J$, i.e. $g \in N_{G}(J)$. Hence, $J=\langle A, B\rangle$ is $\mathfrak{F}$-weakly normal in $G$ as claimed.

\section{Proofs of the main results.}

Proof of Corollary 1. Apply Theorem 1(i) and Lemma 2(iii).

Proof of Corollary 2. One direction is clear from Theorem 2. For the converse, we know $G$ satisfies condition (ii) of Theorem 2, so with $X=G$ we obtain $G^{\mathfrak{F}}=G^{\mathfrak{N}}$. Then, because $G$ is a $P S T$-group, conditions (i) and (iii) are held by [2, Theorem 2.1.8]. Applying Theorem 2 yields the result.

Proof of Corollary 3. We need only to prove that if $H$ is pronormal in $G \in f T_{\mathfrak{F}} \cap \mathfrak{S}$, then $H$ is $\mathfrak{F}$-pronormal in $G$. Note that by Corollary 1 , if $T \leq G$, then $T$ is also an $f T_{\mathfrak{F}}$-group. Then by Theorem 2(ii), $T / T^{\mathfrak{F}}$ is nilpotent, so $T^{\overline{\mathfrak{F}}}=T^{\mathfrak{N}}$. Thus, if $H$ is pronormal in $G$ and $g \in G$, let $T=\left\langle H, H^{g}\right\rangle$. Then $H^{g}=H^{x}$ for some $x \in T^{\mathfrak{N}}=T^{\mathfrak{F}}$, and $H$ is $\mathfrak{F}$-pronormal in $G$.

Proof of Theorem 3. Suppose $G$ is a soluble $T_{\mathfrak{F}}$-group. As remarked above, $G$ is a $T$ group. Now if $U$ is a subgroup of $G$ and $P$ is a Sylow $p$-subgroup of $U$, either $P \leq G^{\mathfrak{N}}$, and hence $P$ is normal in $G$, or $P$ is a Sylow $p$-subgroup of the normal subgroup $P G^{\mathfrak{N}}$ of $G$ by Theorem 2. Thus, $U$ is normally embedded in $G$. In particular, $U$ is pronormal in $G$ by [8, I, Theorems (7.13), (6.14)]. Then by Corollary 3, every subgroup of $G$ is $\mathfrak{F}$-pronormal in $G$, establishing that (i) implies (ii).

Lemma 5 immediately yields that (ii) implies (iii).

To prove that (iii) implies (i), let $G$ be a group of minimal order such that every subgroup of $G$ is $\mathfrak{F}$-weakly normal in $G$, but $G \notin T_{\mathfrak{F}} \cap \mathfrak{S}$. Then every subgroup of $G$ is weakly normal in $G$ by the note following Lemma 4, so $G$ is a soluble $T$-group by [2, Theorem 2.3.1]. We need to only establish that $G \in T_{\mathfrak{F}}$.

Now if $L$ is a subgroup of $G$ and $H$ is a subgroup of $L$, then $H$ is $\mathfrak{F}$-weakly normal in $L$. For, if $x \in L$ and $\left\langle H, H^{x}\right\rangle^{\mathfrak{F}} \leq N_{L}(H)$, then $x \in G$ and $\left\langle H, H^{x}\right\rangle^{\mathfrak{F}} \leq N_{G}(H)$, so by hypothesis $x \in N_{G}(H)$. Therefore, $x \in N_{L}(H)$ as required. Our choice of $G$ then implies that every proper subgroup of $G$ is in $T_{\mathfrak{F}} \cap \mathfrak{S}$. Also, if $N>1$ is normal in $G$ and $H / N$ is a subgroup of $G / N$, then $H / N$ is $\mathfrak{F}$-weakly normal in $G / N$. For, if $x N \in G / N$ and $\left\langle H / N,(H / N)^{x N}\right\rangle^{\mathfrak{F}} \leq N_{G / N}(H / N)$, then by [8, II, Lemma 2.4], $\left(\left\langle H, H^{x}\right\rangle / N\right)^{\mathfrak{F}}=$ $\left\langle H, H^{x}\right\rangle^{\mathfrak{F}} N / N \leq N_{G}(H) / N$, so $\left\langle H, H^{x}\right\rangle^{\mathfrak{F}} \leq N_{G}(H)$. Thus, by definition of $\mathfrak{F}$-weakly normal, $x \in N_{G}(H)$, so $x N \in N_{G / N}(H / N)$, as desired. Hence, by choice of $G$, every proper epimorphic image of $G$ is in $T_{\mathfrak{F}} \cap \mathfrak{S}$.

Thus, if $X$ is a proper subnormal subgroup of $G, X \in T_{\mathfrak{F}}$. Then by Theorem 2(ii), $X / X^{\mathfrak{F}}$ is Dedekind, and by Corollary 2, we need to only show that $G / G^{\mathfrak{F}}$ is Dedekind.

First, suppose that $G^{\mathfrak{F}}>1$. Then as seen above, $G / G^{\mathfrak{F}}$ is a soluble $T_{\mathfrak{F}}$-group; it is also in $\mathfrak{F}$, so it is Dedekind. Hence, we may assume $G^{\mathfrak{F}}=1$. Suppose $M$ is maximal in $G$. Now $M$ is $\mathfrak{F}$-weakly normal in $G$ by hypothesis, so $M$ is normal in $G$ by Lemma 6. Hence, the $T$-group $G$ is nilpotent by [8, A, Theorem A(8.3)], so $G$ is Dedekind, as required. 
Note that it is only in (i) implies (ii) that we have to assume more than that $\mathfrak{F}$ contains $\mathfrak{N}$.

Proof of Theorem 4. To establish (i), suppose that every subgroup of $G$ is $\mathfrak{F}$-weakly Frattini in $G$, and that $H$ is $\mathrm{K}$-F-subnormal in $G$. Then by definition, $N_{G}(H) H=G$, so $N_{G}(H)=G$ and $H$ is normal in $G$. Hence, $G$ is a $T_{\mathfrak{F}}$-group.

For (ii), suppose that $G$ is a soluble $T_{\mathfrak{F}}$-group and $H$ is a subgroup of $G$ contained in a K-F-subnormal subgroup $L$ of $G$. Because $G$ is a $T_{\mathfrak{F}}$-group, $L$ is actually normal in $G$, and by Theorem $3, H$ is $\mathfrak{F}$-pronormal in $G$. Thus, $H$ is pronormal in $G$, implying that $H$ satisfies the weak Frattini argument in $G$. Hence, $N_{G}(H) L=G$, as required. The converse of the equivalence stated in (ii) follows immediately from (i).

Proof of Theorem 5. Suppose first that $G$ is a soluble $P S T_{\mathfrak{F}}$-group. Then $G$ is a soluble $P S T$-group, so if $H$ is normal in a Sylow $p$-subgroup of $G$, then $H$ is pronormal in $G$. Then by Corollary $3, H$ is $\mathfrak{F}$-pronormal in $G$, so $G$ satisfies $\mathbf{H}_{p \mathfrak{F}}$. By Corollary 1 , every subgroup of $H$ is a $P S T_{\mathfrak{F}}$-group, so it too satisfies $\mathbf{H}_{p \mathfrak{F}}$.

Now suppose that $G$ is a group of minimal order such that every subgroup of $G$ satisfies $\mathbf{H}_{p \mathfrak{F}}$ for all primes $p$, but $G$ is not a $P S T_{\mathfrak{F}}$-group. Note that every subgroup of $G$ satisfies $\mathbf{H}_{p}$ for all primes $p$, so $G$ is a soluble $P S T$-group. Then by Corollary 2, we need to only show that $X / X^{\mathfrak{F}}$ is nilpotent for every subnormal subgroup $X$ of $G$. Now if $X<G, X$ is a $P S T_{\mathfrak{F}}$-group by minimality of $G$, so $X / X^{\mathfrak{F}}$ is nilpotent by Theorem 2(ii). Thus, we need to only show that $G / G^{\mathfrak{F}}$ is nilpotent.

Suppose $N=G^{\mathfrak{F}}>1$. The proof that for each $p, \mathbf{H}_{p \mathfrak{F}}$ is preserved under the taking of homomorphic images can be derived from the proof of [2, Lemma 2.2.22] using Lemma 3(ii) and (iii). Thus, by induction, $G / N$ is a $P S T_{\mathfrak{F}}$-group, and it is in $\mathfrak{F}$, so by Lemma 2(iv), $G / N$ is nilpotent, as claimed. Thus, we may assume $N=1$ so that $G \in \mathfrak{F}$.

Now suppose $L$ is a non-trivial minimal normal subgroup of $G$ so that, as observed above, $G / L$ is a $P S T_{\mathfrak{F}}$-group in $\mathfrak{F}$, and therefore is nilpotent. Then because $\mathfrak{N}$ is saturated, $G$ is primitive, with $L$ being the unique minimal normal subgroup of $G$, which is a self-centralising Hall subgroup of $G$, and with $M$ being a core-free maximal subgroup complementing $L$ in $G$. Because $G$ is a $P S T$-group, by [2, Theorem 2.1.8] $L$ is of prime order $p$. Let $q \neq p$ be a prime dividing $|M|$, and let $Q$ be a subgroup of order $q$ in the centre of the Sylow $q$-subgroup of $M$, which is a Sylow $q$-subgroup of $G$. Then by hypothesis, $Q$ is $\mathfrak{F}$-pronormal in $G$. Now $Q$ acts faithfully on $L$ because $C_{M}(L)=1$, so $L Q \in \mathfrak{X}_{(p, q)}$, and $L Q$, being normal in $G=L M$ because $M$ is nilpotent, is an element of $\mathfrak{F}$. But then if $x$ is a generator of $L,\left\langle Q, Q^{x}\right\rangle^{\mathfrak{F}}=(L Q)^{\mathfrak{F}}=1$, so $Q$ and $Q^{x}$ are not conjugate by an element of $\left\langle Q, Q^{x}\right\rangle^{\mathfrak{F}}$, contradicting the $\mathfrak{F}$-pronormality of $Q$ in $G$ and establishing the result.

Proof of Corollary 4. One direction follows immediately from Corollaries 1 and 3 , so suppose $G$ is a soluble $P S T$-group such that in every subgroup $L$ of $G$, every pronormal subgroup of $L$ is $\mathfrak{F}$-pronormal in $L$. Then we know that for every prime $p$, every subgroup of $G$ satisfies $\mathbf{H}_{p}$. Thus, if $J$ is normal in a Sylow $p$-subgroup of $L, J$ is pronormal in $L$, and hence $J$ is $\mathfrak{F}$-pronormal in $L$ by hypothesis. This means that $L$ satisfies $\mathbf{H}_{p \mathfrak{F}}$ for all primes $p$, and Theorem 5 yields $G \in P S T_{\mathfrak{F}}$.

Proof of Corollary 5. We begin with the $P T$-group case: Let $G$ be a soluble $P T_{\mathfrak{F}^{-}}$ group. By Corollary 1, any subgroup $L$ of $G$ is also a soluble $P T_{\mathfrak{F}}$-group, so $L$ is a soluble $P T$-group; hence by [2, Theorems 2.2.3, 2.2.25], for any prime $p, L$ satisfies $\mathbf{H}_{p}$ 
and has Iwasawa Sylow $p$-subgroups. But by Corollary 3, any pronormal subgroup of $L$ is $\mathfrak{F}$-pronormal in $L$, so $L$ also satisfies $\mathbf{H}_{p \mathfrak{F}}$.

Now let $G$ be a group of minimal order such that $G$ has Iwasawa Sylow $p$-subgroups and satisfies $\mathbf{H}_{p \mathfrak{F}}$ for all primes $p$, but $G \notin P T_{\mathfrak{F}}$. Then for each $p, G$ satisfies $\mathbf{H}_{p}$ and has Iwasawa Sylow $p$-subgroups, so $G$ is a soluble $P T$-group by [2, Theorems 2.2.3, 2.2.25].

Suppose first that $G \in \mathfrak{F}$, and let $K$ be a non-trivial normal subgroup of $G$. Then $G / K$ has Iwasawa Sylow $p$-subgroups because $G$ does, and, as seen above, $G / K$ satisfies $\mathbf{H}_{p \mathfrak{F}}$ for all primes $p$. Thus, $G / K \in P T_{\mathfrak{F}}$, so $G / K \in p W$ by Lemma 2(iv). In particular, $G / K \in \mathfrak{N}$. Thus, if $K$ is not a unique minimal normal subgroup of $G$, then $G \in \mathfrak{N}$ as in the proof of Theorem 5, so every subgroup of the $P T$-group $G$ is subnormal in $G$, and therefore permutable in $G$, i.e. $G \in p W$, so $G \in P T_{\mathfrak{F}}$ as claimed. We now proceed just as in the proof of Theorem 5 to obtain a contradiction.

Thus, we may assume $G^{\mathfrak{F}}>1$. Then for all $p, G / G^{\mathfrak{F}}$ satisfies $\mathbf{H}_{p \mathfrak{F}}$ and has Iwasawa Sylow $p$-subgroups since $G$ does, so by minimality of $G, G / G^{\mathfrak{F}}$ is a soluble $P T_{\mathfrak{F}^{-}}$ subgroup. Therefore, Lemma 2(iv) implies that $G / G^{\mathfrak{F}} \in p W$, so $G^{\mathfrak{F}}=G^{\mathfrak{N}}$.

Now by Theorem 1(i), $G$ is minimal with respect to having Iwasawa Sylow $p$ subgroups and satisfying $\mathbf{H}_{p \mathfrak{F}}$ for all $p$, but with $G \notin p R_{\mathfrak{F}}$. Thus, since $G$ is a soluble $P T$-group that is not in $p R_{\mathfrak{F}}$, it has a section $A / B$ that is isomorphic to some $X_{(t, q)} \in$ $\mathfrak{X}_{\mathfrak{F}}$.

Note that because $A$ is not $t^{\prime}$-closed, $A \cap O_{t^{\prime}}(G)=1$, so $G / O_{t^{\prime}}(G)$ has a section isomorphic to $A / B$, has Iwasawa Sylow $p$-subgroups and satisfies $\mathbf{H}_{p \mathfrak{F}}$ for all primes $p$, so by minimality of $G, O_{t^{\prime}}(G)=1$. Now we can choose a Hall $(t, q)$-subgroup $T Q$ of $G$, where $T$ and $Q$ are, respectively, a Sylow $t$-subgroup and Sylow $q$-subgroup of $G$ such that $T \cap A$ and $Q \cap A$ are Sylow subgroups of $A$ and $T Q \cap A$ is a Hall subgroup of $A$. Then since $A / B$ is isomorphic to a homomorphic image of $T Q \cap A$, some section of $T Q$ is isomorphic to $X_{(t, q)}$, so $T Q \notin p R_{\mathfrak{F}}$. But $T Q$, being a subgroup of a soluble $P T$-group, is a $P T$-group, and its Sylow subgroups are Iwasawa because they are Sylow subgroups of $G$. For the same reason if $H$ is a normal subgroup of a Sylow subgroup of $T Q$, it is a normal subgroup of a Sylow subgroup of $G$, so it is $\mathfrak{F}$-pronormal in $G$, and by Lemma 3(i), $H$ is $\mathfrak{F}$-pronormal in $T Q$. Thus, $T Q$ satisfies $\mathbf{H}_{p \mathfrak{F}}$ for all $p$. Thus, by minimality of $G, G=T Q$.

By [2, Theorem 2.1.8], $G^{\mathfrak{N}}$ is a normal abelian Hall subgroup of $G$, so since it is non-trivial and $O_{t^{\prime}}(G)=1, G^{\mathfrak{N}}=T$, and by [2, Theorem 2.1.8], every subgroup of $T$ is normal in $G$. Then suppose $t$ divides $|B|$, and let $C$ be the Sylow $t$-subgroup of $B$, so $C \leq T$ and $C$ is normal in $G$. Then $G / C$ still has Iwasawa Sylow $p$-subgroups and satisfies $\mathbf{H}_{p \mathfrak{F}}$ for all $p$, and $(A / C) /(B / C)$ is isomorphic to $X_{(t, q)}$, so by minimality of $G, C=1$, a contradiction. Thus, $B$ is a $q$-group, and $|A|=t q|B|$, so if $D$ is a Sylow $t$-subgroup of $A$, then $|D|=t$, and $D \leq T$, so $D$ is normal in $G$; thus $D$ is normal in $T$ and is of prime order, so $D \leq Z(T)$. And $B$ is in every Sylow $q$-subgroup of $A$, so it is contained in $Q$. Since both $B$ and $D$ are normal in $A, B$ centralises $D$. But $D$ is a chief factor of $G$ below $T$, and by [2, Lemma 2.1.3], all chief factors of $G$ below $T$ are $G$-isomorphic, so $B$ centralises all of them. Because $|B|$ is relatively prime to $|T|, B$ centralises $T$, so $B \leq C_{Q}(T)$, which is normal in $Q$ because $T$ is normal in $G$. Hence, since it centralises $T, C_{Q}(T)$ is normal in $G$. But $O_{t^{\prime}}(G)=1$, so $C_{Q}(T)=1$, implying that $B=1$ and $A \in \mathfrak{X}_{(t, q)}$.

Let $E$ denote the Sylow $q$-subgroup $Q \cap A$ of $A$. Then $A=D E$, and $E$ does not centralise $D$. Thus, if $T_{1}$ is a subgroup of $T$ of index $t, E$ acts non-trivially on $T / T_{1}$. This implies that $G / T_{1}$ has a section isomorphic to $X_{(t, q)}$, so as above, by minimality 
of $G, T_{1}=1$ and $|T|=t$, so $D=T$. Then $C_{Q}(T)=1$ implies that $Q$ is cyclic of order dividing $t-1$. This means $E$ is normal in $Q$, so by $\mathbf{H}_{p \mathfrak{F}}, E$ is $\mathfrak{F}$-pronormal in $G$, and therefore $E$ is $\mathfrak{F}$-pronormal in $A$. Now $A \in \mathfrak{X}_{(t, q)}$, and $T \leq A$ implies $A$ is subnormal in $G$, so $A \in \mathfrak{F}$ by property $\alpha$. But $E$ is maximal in $A$, so by Lemma 3(iv), $E$ is normal in $A$ and therefore centralises $D$, the final contradiction.

To see that a similar proof works for $T$-groups, we observe that by [2, Theorems 2.2.2, 2.2.3 and 2.2.4] the characterisation of soluble $P T$-groups can be changed to a characterisation of $T$-groups simply by replacing 'Iwasawa' by 'Dedekind': A group is a soluble $T$-group if and only if for all primes $p$ it satisfies $\mathbf{H}_{p}$ and has Dedekind Sylow $p$-subgroups. Because Corollary 3 and Theorem 1 apply to $f=n$ as well as $f=p$, the proof above carries through by changing 'Iwasawa' to 'Dedekind', ' $P T$ ' to ' $T$ ' and ' $p$ ' to ' $n$ '.

Proof of Corollary 6. For (i), note that if $G$ is a soluble $P T_{\mathfrak{F}}$-group and $L$ is a subgroup of $G$, then $L$ is a soluble $P T_{\mathfrak{F}}$-group. Thus, by Corollary 3 , every pronormal subgroup of $L$ is $\mathfrak{F}$-pronormal in $L$. Conversely, suppose $G$ is a soluble $P T$-group such that each pronormal subgroup of $G$ is $\mathfrak{F}$-pronormal in $G$. Then for each prime $p, G$ has Iwasawa Sylow $p$-subgroups. Suppose $H$ is normal in a Sylow $p$-subgroup of $G$. Now $G$ is a soluble $P T$-group, so $G$ satisfies $\mathbf{H}_{p}$. Thus, $H$ is pronormal in $G$, so by hypothesis $H$ is $\mathfrak{F}$-pronormal in $G$. Hence, $G$ satisfies $\mathbf{H}_{p \mathfrak{F}}$. Thus, by Corollary $5, G \in P T_{\mathfrak{F}}$.

The proof of (ii) is analogous.

Proof of Theorem 7. First, we know that if $G \in f T_{\mathfrak{F}}$, then $H$ K- $\mathfrak{F}$-sn $G$ implies $H$ $f G$, so $G \in f T_{p \mathfrak{F}}$ for all primes $p$.

For the converse, first we prove that $f T_{p \mathfrak{F}}$ is closed under the taking of epimorphic images and subnormal subgroups. So let $N$ be normal in $G \in f T_{p \mathfrak{F}}$, and suppose $H / N$ is a $p^{\prime}$-perfect $\mathrm{K}-\mathfrak{F}$-subnormal subgroup of $G / N$. Then $H \mathrm{~K}-\mathfrak{F}$-sn $G$ by [4, Lemma 2(ii)], so $O^{p^{\prime}}(H) \mathrm{K}$ - - -sn $G$. Since $O^{p^{\prime}}(H)$ is $p^{\prime}$-perfect, $O^{p^{\prime}}(H) f G$ by hypothesis, so $H / N=O^{p^{\prime}}(H / N)=O^{p^{\prime}}(H) N / N f G / N$. Thus, $G / N \in f T_{p \mathfrak{F}}$, as claimed. Next, let $X$ be a subnormal subgroup of $G \in f T_{p \mathfrak{F}}$. Then if $H \mathrm{~K}-\mathfrak{F}$-sn $X$ and $H$ is $p^{\prime}$-perfect, then $H \mathrm{~K}-\mathfrak{F}$-sn $G$ and $H$ is $p^{\prime}$-perfect, so $H f G$, implying $H f X$ because $X$ sn $G$. Thus, $X \in f T_{p \mathfrak{F}}$, as claimed.

Then suppose $\mathfrak{F}$ has property $\alpha$, and $G$ is a minimal soluble group such that $G \in f T_{p \mathfrak{F}}$ for all $p$, but $G \notin f T_{\mathfrak{F}}$. First, $G \in f T_{p}$ for all $p$, so $G \in f T$ by Theorem 6 . Thus, by Corollary 2 we need only show that $X / X^{\mathfrak{F}} \in f W$ for all subnormal subgroups $X$ of $G$. Then if $X$ sn $G$ and $X<G$, by minimality of $G, X \in f T_{\mathfrak{F}}$, so $X / X^{\mathfrak{F}} \in f W$ by Lemma 2(iv). Now if $G \notin \mathfrak{F}, G / G^{\mathfrak{F}} \in f T_{\mathfrak{F}}$ by minimality of $G$ and $G / G^{\mathfrak{F}} \in f W$. So suppose $G \in \mathfrak{F}$. Let $P$ be a Sylow $p$-subgroup of $G$. Then by Lemma 2(iv), $P$ K-F- $-s n$ $G$, and clearly $P$ is $p^{\prime}$-perfect, so $P f G$ by hypothesis. Hence, $P$ sn $G$, and therefore $P$ is normal in $G$. Hence, $G$ is nilpotent. So every subgroup of $G$ is subnormal in $G$, and $G \in f T$, so every subgroup of $G$ is in $f(G)$. Thus, $G \in f W$ as claimed, and by Corollary $2, G \in f T_{\mathfrak{F}}$.

ACKNOWLEDGEMENTS. The first author has been supported by the grant MTM2010-19938-C03-01 from MICINN (Spain) and Project of NSFC (11271085). The third author wishes to thank Franklin \& Marshall College for its generous sabbatical policy, and National University of Ireland, Galway and Universitat de València and Universitat Politècnica de València for their support. 


\section{REFERENCES}

1. A. Ballester-Bolinches, J. C. Beidleman, A. D. Feldman and M. F. Ragland, On generalised subnormal subgroups of finite groups, Math. Nachr. 286(11-12) (2013), 1066-1071.

2. A. Ballester-Bolinches, R. Esteban-Romero and M. Asaad, Products of finite groups, De Gruyter Expositions in Mathematics, vol. 53 (Springer, Berlin, Germany, 2010) 584 pp.

3. A. Ballester-Bolinches and L. M. Ezquerro, Classes of finite groups, Mathematics and Its Applications (Springer, Berlin, Germany, 2006), 584 pp.

4. A. Ballester-Bolinches, A. D. Feldman, M. C. Pedraza-Aguilera and M. F. Ragland, A class of generalised finite T-groups, J. Algebra 333(1) (2011), 128-138.

5. A. Ballester-Bolinches and M. D. Pérez-Ramos, On $\mathfrak{F}$-subnormal subgroups and Frattini-like subgroups of a finite group, Glasgow Math. J. 36 (1994), 241-247.

6. J. C. Beidleman and H. Heineken, Finite soluble groups whose subnormal subgroups permute with certain classes of subgroups, J. Group Theory 6 (2003), 139-158.

7. R. A. Bryce and J. Cossey, The Wielandt subgroup of a finite soluble group, J. London Math. Soc. 40 (1989), 244-256.

8. K. Doerk and T. Hawkes, Finite soluble groups (De Gruyter, Berlin, Germany, 1992).

9. A. Feldman, $\mathfrak{F}$-Bases and subgroup embedding in finite solvable groups, Arch. Math. 47 (1986), 481-492.

10. A. D. Feldman, t-groups and their generalizations, Group theory (Granville, $\mathrm{OH}, 1992)$ (World Sci. Publ., River Edge, NJ, 1993), 128-133.

11. N. Müller, $\mathfrak{F}$-pronormale untergruppen endlich aufösbarer gruppen. (Johannes Gutenberg-Universität Mainz, Diplomarbeit, 1985).

12. T. A. Peng, Finite groups with pro-normal subgroups, Proc. Amer. Math. Soc. 20 (1969), 232-234.

13. D. J. S. Robinson A note on finite groups in which normality is transitive, Proc. Amer. Math. Soc. 19 (1968), 933-937. 